\title{
The velocity field of sunspot penumbrae
}

\section{A global view}

\author{
M. Franz and R. Schlichenmaier
}

Kiepenheuer Institut für Sonnenphysik, Schöneckstraße 6, 79104 Freiburg, Germany

e-mail: morten@kis.uni-freiburg.de

Received 6 August 2009 / Accepted 24 September 2009

ABSTRACT

\begin{abstract}
Aims. We investigated the vertical penumbral plasma flow on small spatial scales using data recorded by the spectropolarimeter of the solar optical telescope onboard Hinode.

Methods. We computed maps of apparent Doppler velocities by comparing the spectral positions of the Fe I $630.15 \mathrm{~nm} \&$ Fe I $630.25 \mathrm{~nm}$ lines with the averaged line profiles of the quiet Sun. To visualize the flow pattern in the low photosphere, we used a bisector of the wing of the absorption lines. The small heliocentric angle $\left(3^{\circ} \leq \Theta \leq 9^{\circ}\right)$ of our data sets means that the horizontal component of the Evershed flow (EF) does not contribute significantly to the line shift.

Results. We found that in the quiet Sun (QS), the area showing upflows is always larger than the one exhibiting downflows. In the penumbra, upflows dominate only at low velocities $\left|v_{\text {dop }}\right| \leq 0.4 \mathrm{~km} \mathrm{~s}^{-1}$, while at higher velocities $\left|v_{\text {dop }}\right| \geq 0.6 \mathrm{~km} \mathrm{~s}^{-1}$ downflows prevail. Additionally, the maximal upflow velocity in penumbrae is lower, while the maximal downflow velocity is larger with respect to the QS velocities. Furthermore, on a spatial average, the penumbra shows a redshift, corresponding to a downflow of more than $0.1 \mathrm{~km} \mathrm{~s}^{-1}$. Upflows are elongated and appear predominately in the inner penumbra. Strong downflows with velocities of up to $9 \mathrm{~km} \mathrm{~s}^{-1}$ are concentrated at the penumbra-QS boundary. They are magnetized and are rather round. The inner penumbra shows an average upflow, which turns into a mean downflow in the outer penumbra. The upflow patches in the inner penumbra and the downflow locations in the outer penumbra could be interpreted as the sources and the sinks of the EF. We did not find any indication of roll-type convection within penumbral filaments.
\end{abstract}

Key words. sunspots - Sun: photosphere

\section{Introduction}

Although penumbrae of sunspots have been subject to scientific investigation for a long time, there remain a lot of open questions regarding e.g. their filamentary fine structure, the energy transport, and the plasma dynamics on scales of less than $1^{\prime \prime}$. The gappy model (Spruit \& Scharmer 2006) assumes the existence of field-free, radially aligned gaps below the $\tau=1$ level intruding into a potential field above. Dark cores of penumbral filaments (Scharmer et al. 2002) are explained as a variation of the $\tau=1$ level across the tip of the field-free gap, which is similar to the explanation of dark lanes in umbral dots given by Schüssler \& Vögler (2006). Even though the gappy model provides an explanation not only for the dark cores but also for the brightness of the penumbra, it poses a problem because it does not explain the magnetized Evershed flow (EF) (Rezaei et al. 2006).

In the uncombed model (Solanki \& Montavon 1993), nearly horizontal flux tubes are assumed to be embedded in a less inclined magnetic background field (Schlichenmaier et al. 1998a,b). Strong gradients of atmospheric parameters are encountered for any line of sight (LOS) penetrating both the background atmosphere and the flux tube. It has recently been shown that dark cores of penumbral filaments can be explained within the framework of this model by taking the hot EF, embedded in a stratified atmosphere into account (Ruiz Cobo \& Bellot Rubio 2008). Despite the success of the uncombed model in explaining e.g. the EF, as well as the existence of bright filaments and their migration, the permanent stability of magnetic flux tubes in the penumbral photosphere is questionable.
Recently, significant progress has been made with simulations of entire sunspots. Different 3D MHD codes were used to model sunspots in slab geometry (Heinemann et al. 2007) or even the entire spot at once (Rempel et al. 2009), which reproduces a variety of observed features, e.g. dark-cored penumbral filaments and their migration. To either endorse or disprove these results and to gain a deeper understanding of the convective nature of the energy transport in the inclined magnetic field of the penumbra, it is thus crucial to investigate the plasma flow in the low photosphere on scales of less than 1 ".

So far, velocities are either inferred from inversion (e.g. SIR, Milne-Eddington) (Bellot Rubio et al. 2007; Scharmer et al. 2008) or magnetograms that are constructed in the far wings of magnetic absorption lines, thereby identifying strongly blueor red-shifted polarization signals (Ichimoto et al. 2007). The strength of the signal in the far wing, however, does not depend only on the velocity itself, but also on field inclination or gas temperature (Bellot Rubio 2009).

In this contribution, we present measurements of line shifts in the wings of the Stokes $I$ profile, which enables us to analyze the Doppler velocities, directly and quantitatively.

\section{Observation}

We used data obtained by the spectropolarimeter (SP) (Lites et al. 2001) of the solar optical telescope (SOT) (Tsuneta et al. 2008) onboard the Hinode satellite. The SP records the Stokes spectra of the two iron lines at $630.15 \mathrm{~nm}$ and $630.25 \mathrm{~nm}$, with 
Table 1. Data samples used in this study.

\begin{tabular}{cccc}
\hline \hline Name & $\begin{array}{c}\text { NOAA } \\
\text { Active region }\end{array}$ & $\begin{array}{c}\text { Date of } \\
\text { observation }\end{array}$ & $\begin{array}{c}\text { Heliocentric angle } \\
\cos (\Theta)\end{array}$ \\
\hline Spot A & 10923 & Nov. 14th 2006 & $0.982-0.996$ \\
Spot B & 10923 & Nov. 14th 2006 & $0.980-0.994$ \\
Spot C & 10930 & Dec. 11th 2006 & $0.991-0.999$ \\
Spot D & 10933 & Jan. 05th 2007 & $0.996-1.000$ \\
QS 1 & $\ldots$ & Mar. 10th 2007 & $0.970-1.000$ \\
QS 2 & $\ldots$ & Sep. 06th 2007 & $0.988-1.000$ \\
\hline
\end{tabular}

Landé factors of $g=1.67$ and $g=2.5$, respectively. By scanning the spectrograph slit across the target in steps of 0.15 and achieving a wavelength sampling of $2.15 \mathrm{pm} / \mathrm{pixel}$, twodimensional maps of the field of view (FOV) were obtained. Since the width of the slit is equivalent to $0{ }^{\prime} 16$ and the pixel size along the slit is 0.16 on average, normal SP scans provide a spatial resolution of 0.'32 (Centeno et al. 2009). For an exposure time of $4.8 \mathrm{~s}$ per slit position, the $1 \sigma$ noise level in the Stokes spectra is around $10^{-3} \cdot I_{\mathrm{c}}$, where $I_{\mathrm{c}}$ is the continuum intensity.

For our study, we obtained the raw data summarized in Table 1 and reduced it, using the IDL routine "sp_prep.pro" provided with the "Solar Soft" package. From the Stokes spectra, we computed maps of continuum intensity, $I_{\mathrm{c}}$, total polarization $P_{\text {tot }}=\int\left[\left(Q^{2}+U^{2}+V^{2}\right) / I_{\mathrm{c}}^{2}\right]^{1 / 2} \mathrm{~d} \lambda$, and Doppler velocity along the LOS, $v_{\text {dop }}$. Furthermore, we increased the contrast of the maps of $I_{\mathrm{c}}$ and $P_{\text {tot }}$ by applying a standard IDL high-pass filter to the pictures and then convolved the result with the original. As a consequence, the picture appears sharper, and it becomes easier to distinguish between bright and dark features in the penumbra (e.g. the lateral brightening and the dark core of a filament). While the calculation of the maps of $I_{\mathrm{c}}$ and $P_{\text {tot }}$ is straightforward, the derivation of $v_{\mathrm{dop}}$ requires some additional explanation.

\section{Method}

Since Hinode spectra lack telluric lines, it is impossible to use them to calibrate the velocity scale absolutely. To overcome this problem, we used an average QS profile for calibration purposes. The disadvantage of this method is, however, that the respective lines are displaced from laboratory wavelength by several effects, including the convective blueshift (CBS) (Dravins 1982). Beck (2006) used the SIR code with a two-component model atmosphere of Borrero \& Bellot Rubio (2002) to determine the CBS at disk center. The corresponding velocities of the line core of the average QS profile of Fe I 630.15 and Fe I 630.25 were calculated to be $-185 \mathrm{~m} \mathrm{~s}^{-1}$ and $-262 \mathrm{~m} \mathrm{~s}^{-1}$, respectively (negative velocity corresponds to blueshift).

\subsection{Velocity calibration using Quiet Sun profiles}

We have argued that the line core of an average Stokes $I$ profile of the QS is suitable for an absolute wavelength calibration, if it is corrected for the CBS. However, two effects, the moat flow and the influence of the magnetic field, have to be considered in the surroundings of sunspots.

Because of the residual heliocentric angle, the radially outward oriented moat flow (Vargas Domínguez et al. 2008) would lead to a systematic offset in the calibration, if only the Stokes $I$ profiles of one side of the spot vicinity were taken into account. Likewise, a magnetic field lifts the degeneracy of the atomic

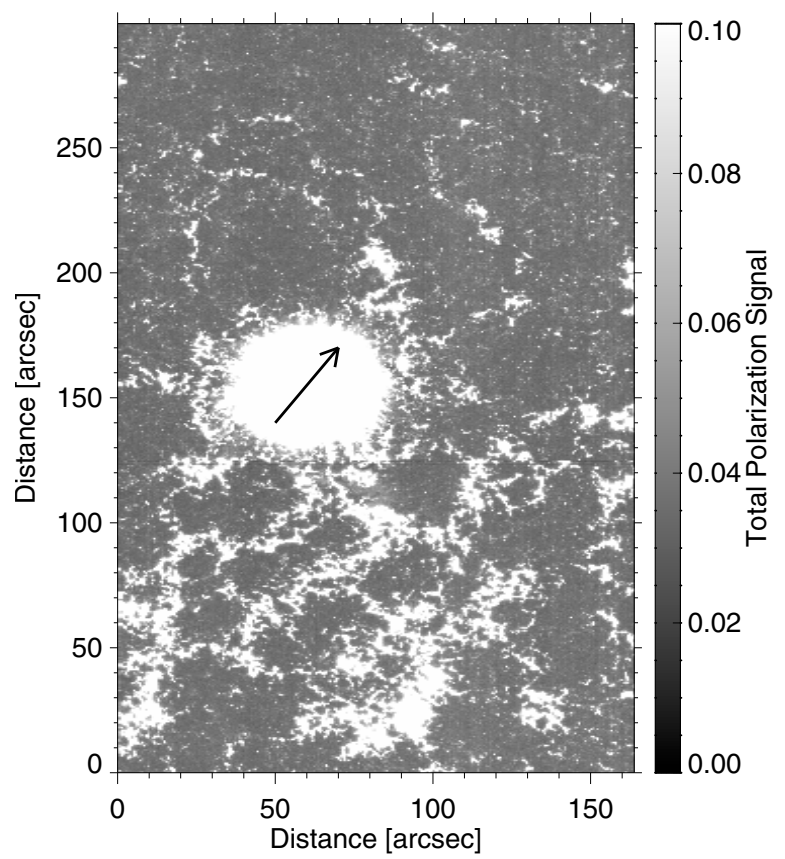

Fig. 1. Total polarization of data sample Spot D. The spectra of the pixels marked in white were not considered for an averaged Stokes $I$ profile of the QS. The arrow points towards disk center at $(x, y) \sim\left(87^{\prime \prime}, 195^{\prime \prime}\right)$.

levels, which causes the profile to split and makes it difficult to determine the position of the line core correctly (Solanki 1993).

Therefore, we decided to average all profiles of the QS surrounding the sunspot, but to exclude those profiles that exhibit a split. To meet this requirement, we created a mask that omits the entire spot and all pixels with significant magnetic field strength. For these purposes, we used $P_{\text {tot }}$ as a proxy and defined a cut-off criterion $P_{\text {tot }}>0.1 \cdot \max \left(P_{\text {tot }}\right)$ for all pixels whose Stokes $I$ spectra should not be averaged (cf. Fig. 1). Finally, the line minima of Fe I 630.15 and Fe I 630.25 in the average Stokes I profile of the QS are determined by fitting a 2nd order polynomial to the core of the respective line (cf. Fig. 2).

\subsection{Velocity calibration using umbral profiles}

Beckers (1977) showed that there is no vertical plasma motion in umbrae of sunspots at disk center. Consequently, an average umbral profile should be redshifted when compared to the line core of an average QS profile. To substantiate the values given by Beck (2006), we followed a procedure described in Rezaei et al. (2006).

Because of the strength of the magnetic field, the core of umbral Stokes $I$ profiles splits, which makes it difficult to determine the core position correctly. Therefore, we determined the center-position - midways between the two lobes - of umbral Stokes $V$ profiles, which corresponds to the line core of the respective Stokes I profile. This parameter can be calculated with reasonable precision if molecular blends are not too strong and both lobes are well defined. If LTE can also be assumed, and no velocity gradients are present in the line-forming region, e.g. above the umbra, Stokes $V$ profiles are strictly antisymmetric with respect to the center-position (Auer \& Heasley 1978). Thus, the center position of antisymmetric Stokes $V$ profiles defines a frame of rest on the solar surface. 


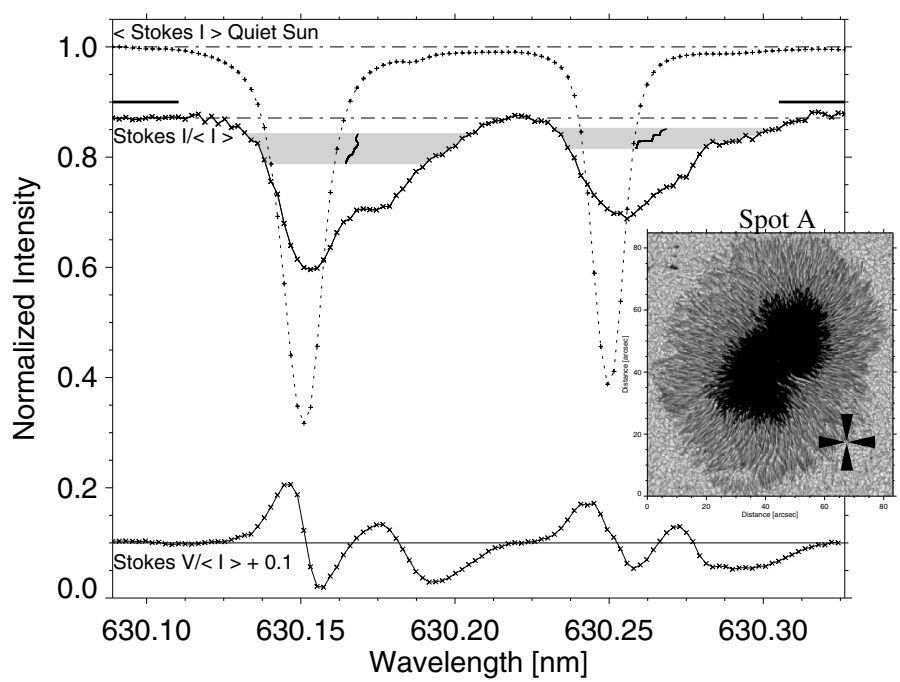

Fig. 2. Stokes $I$ and Stokes $V$ profiles (solid line, measurements plotted with "X" symbols) from a downflow region (marked by the cross hairs in the inlet) in Spot A, as well as the average Stokes $I$ profile from the QS (dotted line, measurements indicated by "+" symbols). The mean value of the bisector (solid) of the line wing (shaded gray) is used to derive the Doppler shift of that pixel. $I_{\mathrm{c}}$ (dashed-dotted) is calculated by averaging the measurements below the two bold lines on the left and right sides of the spectrum.

Table 2. Wavelength calibration using umbral Stokes $V$ profiles.

\begin{tabular}{ccccc}
\hline \hline Name & \multicolumn{2}{c}{ CBS $\left[\mathrm{m} \mathrm{s}^{-1}\right]$} & \multicolumn{2}{c}{$\mathrm{rms}^{a}\left[\mathrm{~m} \mathrm{~s}^{-1}\right]$} \\
& Fe I 630.15 & Fe I 630.25 & Fe I 630.15 & Fe I 630.25 \\
\hline Spot A & -231 & -296 & 93 & 83 \\
Spot B & -245 & -315 & 94 & 82 \\
Spot C & -201 & -295 & 100 & 92 \\
Spot D & -231 & -344 & 147 & 107 \\
\hline
\end{tabular}

${ }^{a}$ These values represent the variation of the center position of umbral Stokes $V$ profiles with $|\delta a|<0.01$.

As a measure for the degree of antisymmetry, we used the amplitude asymmetry. It is defined as the difference of the absolute amplitudes of the two lobes like

$\delta a=\frac{a_{\mathrm{r}}-a_{\mathrm{b}}}{a_{\mathrm{r}}+a_{\mathrm{b}}}$,

with $a_{\mathrm{r}}$ and $a_{\mathrm{b}}$ the amplitude of the red and the blue lobe, respectively. We used a parabola fit around the extrema of both lobes to determine their amplitudes. Following the arguments above, we considered only umbral Stokes $V$ profiles with $|\delta a|<0.01$ and determined their center position. The results are summarized in Table 2.

We find that, in all our data samples the average QS is indeed blueshifted with respect to the average center position of umbral Stokes $V$ profiles obeying $|\delta a|<0.01$. Within the listed rms errors (cf. Table 2), the values for the CBS agree with the ones reported by Beck (2006). Furthermore, we can confirm the applicability of the procedure reported by Rezaei et al. (2006).

\subsection{Flow velocity in the deep photosphere}

As we are interested in the plasma flow in the low photosphere, it is necessary to compute the line position as close to the continuum as possible. On the one hand, the line-forming region of Fe I 630.15 encompasses higher atmospheric layers if compared to Fe I 630.25 , but on the other hand, the lower Landé $\mathrm{g}$ factor means that the former is less sensitive to disturbances by magnetic fields and instrumental crosstalk. In Fe I 630.15, a blend in the red wing close to the continuum obscures the profile and makes it impossible to derive a reliable bisector above $0.9 \cdot I_{\mathrm{c}}$ (cf. Fig. 2). Similar obstacles occur in the spectra of Fe I 630.25 originating from the innermost penumbra. In this area, the magnetic field, hence the line broadening, is so strong that the adjacent iron line serves as a blend, leaving the blue part of the continuum not defined above $0.9 \cdot I_{\mathrm{c}}$. Therefore, we restricted the determination of the bisector of each line to a band of $0.7 \leq I_{\mathrm{c}} \leq 0.9$ (cf. Fig. 2).

Next, we determined the bisector of both lines with subpixel accuracy by interpolating linearly between the spectral measurements. From that bisector, we obtained an average spectral position of the line wing. Any offset between this position and the position of the line core of the averaged QS profile is interpreted as a Doppler shift caused by the velocities of the plasma. Finally, the Doppler maps (cf. Fig. 5) are obtained by computing the velocities that correspond to the Doppler shifts at any given spatial position.

As mentioned above, the low photospheric temperature of the umbra allows the formation of molecules. Their absorption bands mask the two iron lines to an extent where it becomes unreliable for computing a bisector. Therefore, the Doppler velocity of umbral pixels - i.e. pixels obeying $I_{\mathrm{c}}<0.33 \cdot I_{\mathrm{c}}(\mathrm{QS})-$ were manually set to zero (cf. Fig. 5).

\subsection{Error discussion}

The biggest challenge in the calibration process is to determine the line core position of the average QS profile. Due to the finite spectral resolution, we have seven spectral measurements to fit the core. Using, for example, only five measurement to fit the core in sample "Spot A" changes the CBS of the QS by $+40 \mathrm{~m} \mathrm{~s}^{-1}$. In the QS, significant magnetic fields are predominately found in the intergranular downflow regions. As a result, we exclude more pixels showing downflows and introduce a bias to our calibration (cf. Fig. 1). This bias leads to an overall blueshift of the maps between $-10 \mathrm{~m} \mathrm{~s}^{-1}$ and $-30 \mathrm{~m} \mathrm{~s}^{-1}$ in all data samples. The thermal drift in the spectral direction is corrected by the "sp_prep.pro" calibration routine. In all our data samples, it is within the range of $\pm 50 \mathrm{~m} \mathrm{~s}^{-1}$. Furthermore, we have shown that the values of the CBS of the QS inferred from model calculations and the values obtained from measurements differ by no more than $\pm 80 \mathrm{~m} \mathrm{~s}^{-1}$.

Because of the difficulties mentioned above (especially the uncertainties in the position of the line core of the average QS) and since our fit procedure allows us to determine the line core within an accuracy of one tenth of a pixel, we assume a precision of $\pm 0.1 \mathrm{~km} \mathrm{~s}^{-1}$ for our wavelength calibration. Since we do not consider any velocity signal $-0.1 \mathrm{~km} \mathrm{~s}^{-1} \leq v_{\text {dop }} \leq 0.1 \mathrm{~km} \mathrm{~s}^{-1}$ in the following, we are confident that any up- or down-flow is an actual velocity signal.

\section{Results}

\subsection{Differences between Fe I 630.15 and Fe I 630.25}

The resulting velocities derived from the Doppler shifts of the two line wings are comparable: the rms values of the maps deviate less than $0.17 \mathrm{~km} \mathrm{~s}^{-1}$. In general, the maps deduced from the wing of Fe I 630.15 show an overall blueshift with respect to the maps deduced from the wing of Fe I 630.25. 
Table 3. Up- and down-flow velocities in penumbrae \& QS data as derived from Doppler shift of the line wing.

\begin{tabular}{|c|c|c|c|c|c|}
\hline \multirow[t]{2}{*}{ Name } & \multicolumn{2}{|c|}{$v_{\text {dop } 630.15}\left[\mathrm{~km} \mathrm{~s}^{-1}\right]$} & \multicolumn{2}{|c|}{$\left\langle\left\langle v_{\text {dop }}\right\rangle\left[\mathrm{m} \mathrm{s}^{-1}\right]\right.$} & \multirow{2}{*}{$\begin{array}{c}\Delta v_{\mathrm{dop}} \\
\mathrm{rms}\left[\mathrm{m} \mathrm{s}^{-1}\right]\end{array}$} \\
\hline & Up & Down & 630.15 & 630.25 & \\
\hline Spot A & -2.01 & 8.94 & 157 & 126 & 137 \\
\hline Spot B & -2.09 & 6.84 & 158 & 127 & 139 \\
\hline Spot C & -2.24 & 8.88 & 143 & 118 & 168 \\
\hline Spot D & -2.20 & 5.00 & 105 & 100 & 142 \\
\hline QS 1 & -3.09 & 3.15 & -159 & -128 & 83 \\
\hline QS 2 & -3.30 & 3.13 & -114 & -104 & 79 \\
\hline
\end{tabular}

It seems that, in all cases with significant differences $\left(\left|v_{\text {dop } 630.15}-v_{\text {dop }} 630.25\right|>0.5 \mathrm{~km} \mathrm{~s}^{-1}\right)$, the Fe I 630.25 line exhibits Doppler shifts closer to the continuum than the Fe I 630.15 line. This behavior is shown for a selected pixel with a large velocity discrepancy $\left(\Delta v_{\text {dop }}=1.5 \mathrm{~km} \mathrm{~s}^{-1}\right)$ in Fig. 2. Here the Stokes $I$ and Stokes $V$ profiles from a downflow region in the outer penumbra of Spot A are depicted.

Stokes $I$ shows significant differences in the asymmetry of both lines, especially close to the continuum, which has a strong impact on our calculated velocity. In this example, the red wing of Fe I 630.15 is strongly shifted above $0.35 \cdot I_{\mathrm{c}}$. The respective wing of Fe I 630.25, however, shows a strong redshift only above $0.77 \cdot I_{\mathrm{c}}$. Thus, the resulting velocities show significant differences. While the Doppler shift of the average line wing $\left(0.7 \leq I_{\mathrm{c}} \leq 0.9\right)$ of Fe I 630.15 corresponds to $7.8 \mathrm{~km} \mathrm{~s}^{-1}$, the respective Doppler shift of Fe I 630.25 leads to $6.3 \mathrm{~km} \mathrm{~s}^{-1}$, which is a difference of $1.5 \mathrm{~km} \mathrm{~s}^{-1}$. In some cases, the asymmetries are above $0.9 \cdot I_{\mathrm{c}}$ and remain undetected in Fe I 630.25. However, only the amplitude of the velocities, but neither the morphology of up- and down-flow patterns nor the direction of the flow are affected by these deviations.

We do not have any explanation for these differences, but we are tempted to ascribe the variations in the maps of $v_{\text {dop }}$ to differences in the respective line parameters. The line core of Fe I 630.15 forms at $\log \tau=-2.9$, while the core of Fe I 630.25 forms around $\log \tau=-2.0$ (Balthasar 1985) and therefore encompasses lower atmospheric layers. As the strength of the magnetic field decreases with height, its influence on the Fe I 630.25 line is even more significant (Khomenko \& Collados 2007).

\subsection{Global velocity field}

As all data is taken almost at disk center, the maps of Doppler velocities (see Fig. 5 as an example) are dominated by plasma flows normal to the surface. We attribute any asymmetry between the center-side penumbra, which is overall blueshifted, and the limb side, which shows an overall redshift, to a combination of the horizontal EF and projection effects caused by the small heliocentric angle of the respective data sets. The upflow in Spot D appears predominately but not exclusively in the inner penumbra. It encompasses peak velocities around $-2.0 \mathrm{~km} \mathrm{~s}^{-1}$. The downflow shows peak velocities of up to $5.0 \mathrm{~km} \mathrm{~s}^{-1}$, which are generally located at the penumbra-QS boundary, while weaker downflows $\left(0 \mathrm{~km} \mathrm{~s}^{-1} \leq v_{\text {dop }} \leq 2 \mathrm{~km} \mathrm{~s}^{-1}\right)$ appear at all radial distances. The maximal upflow velocities found in the other samples are very similar. The maximum downflow velocity, however, shows variation of up to $80 \%$ (cf. Table 3). Interestingly, the former seems to change on a temporal scale of hours. In Spot A it peaks around $9.0 \mathrm{~km} \mathrm{~s}^{-1}, 9 \mathrm{~h}$ later in Spot B it peaks at $6.9 \mathrm{~km} \mathrm{~s}^{-1}$. On a spatial average the penumbra shows a significant redshift corresponding to the downflow velocity listed in Table 3 .
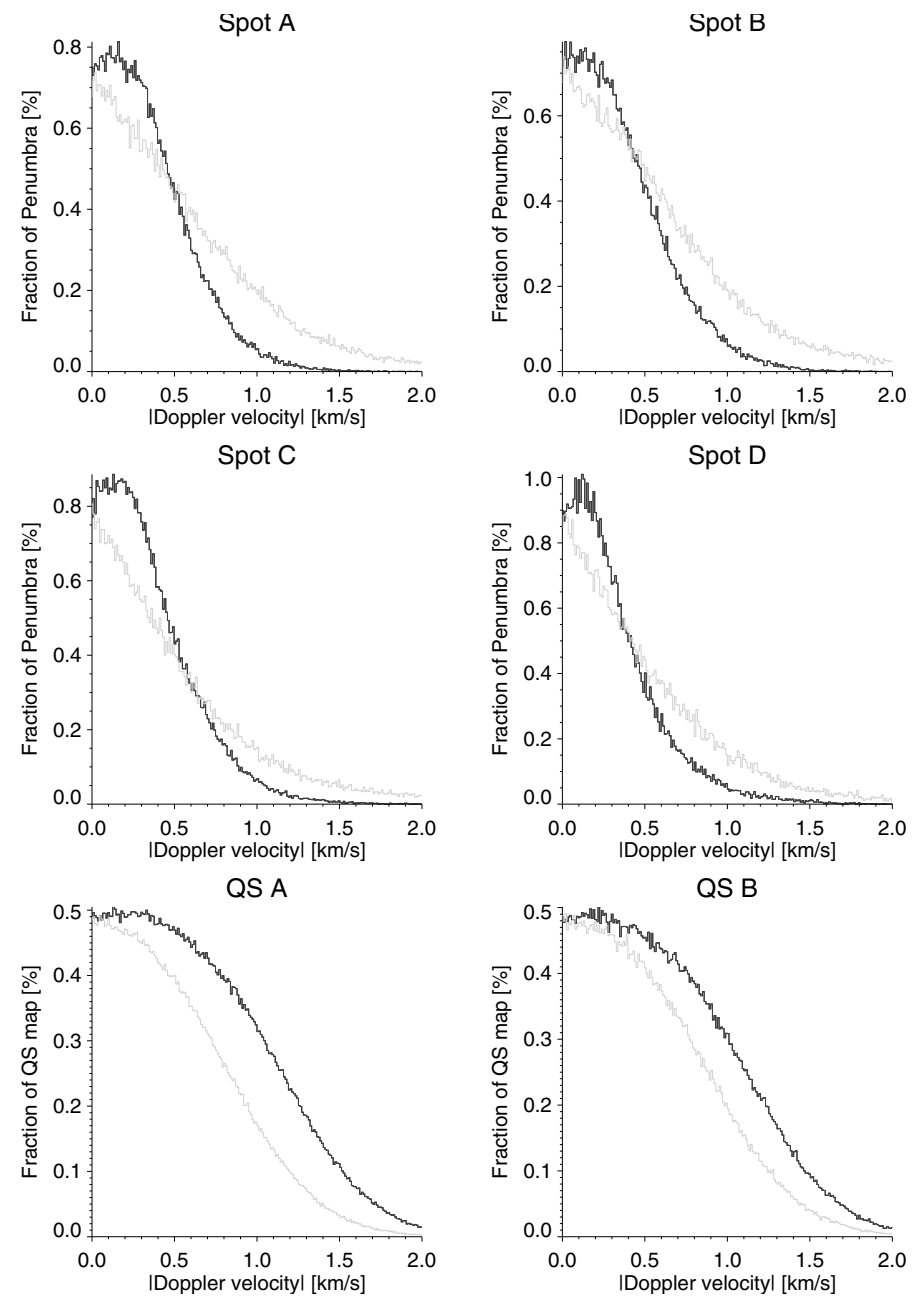

Fig. 3. Histogram of binned Doppler velocities in sunspot (top rows) and QS (bottom row) samples in which the fraction of the penumbra or QS is depicted vs. the absolute value of the Doppler velocity. Black indicates upflow, gray is downflow. In all these measurements, the error is $\pm 0.1 \mathrm{~km} \mathrm{~s}^{-1}$.

The histograms in Fig. 3 show the distribution of velocities in the data sets in more detail. The absolute value of a velocity range of $10 \mathrm{~m} \mathrm{~s}^{-1}$ is binned into one measurement and plotted on the abscissae. The fraction of the penumbra showing the respective velocity range is depicted on the ordinate. Keep in mind that no unambiguous flow direction can be derived for $\left|v_{\text {dop }}\right|<0.1 \mathrm{~km} \mathrm{~s}^{-1}$. For reasons of comparison, QS data is evaluated in the same way (cf. bottom row of Fig. 3).

In all sunspot data, the area occupied by upflows is dominant at low velocities $\left(\left|v_{\text {dop }}\right|<0.4 \mathrm{~km} \mathrm{~s}^{-1}\right)$. The area showing upflows increases slightly and peaks at $-0.1 \leq v_{\text {dop }} \leq-0.2 \mathrm{~km} \mathrm{~s}^{-1}$, which seems to be a preferred velocity for upflows. For higher velocities, this area decreases gradually and is equal in size to the downflow area at velocities $0.4 \leq\left|v_{\text {dop }}\right| \leq 0.6 \mathrm{~km} \mathrm{~s}^{-1}$, but negligible for $v_{\mathrm{dop}} \leq-1.5 \mathrm{~km} \mathrm{~s}^{-1}$. The area showing downflows, in contrast, decreases monotonously towards higher velocities without any maximum and is the dominant one for $\left|v_{\mathrm{dop}}\right|>0.6 \mathrm{~km} \mathrm{~s}^{-1}$. Even at $\left|v_{\text {dop }}\right|>1.5 \mathrm{~km} \mathrm{~s}^{-1}$, there is a significant area of the penumbra occupied with downflows.

In the QS data, the area occupied by upflows is, however, always larger than the area showing downflows, regardless of velocity. Furthermore, the maximum downflow velocity $\left(3 \mathrm{~km} \mathrm{~s}^{-1}\right)$ 


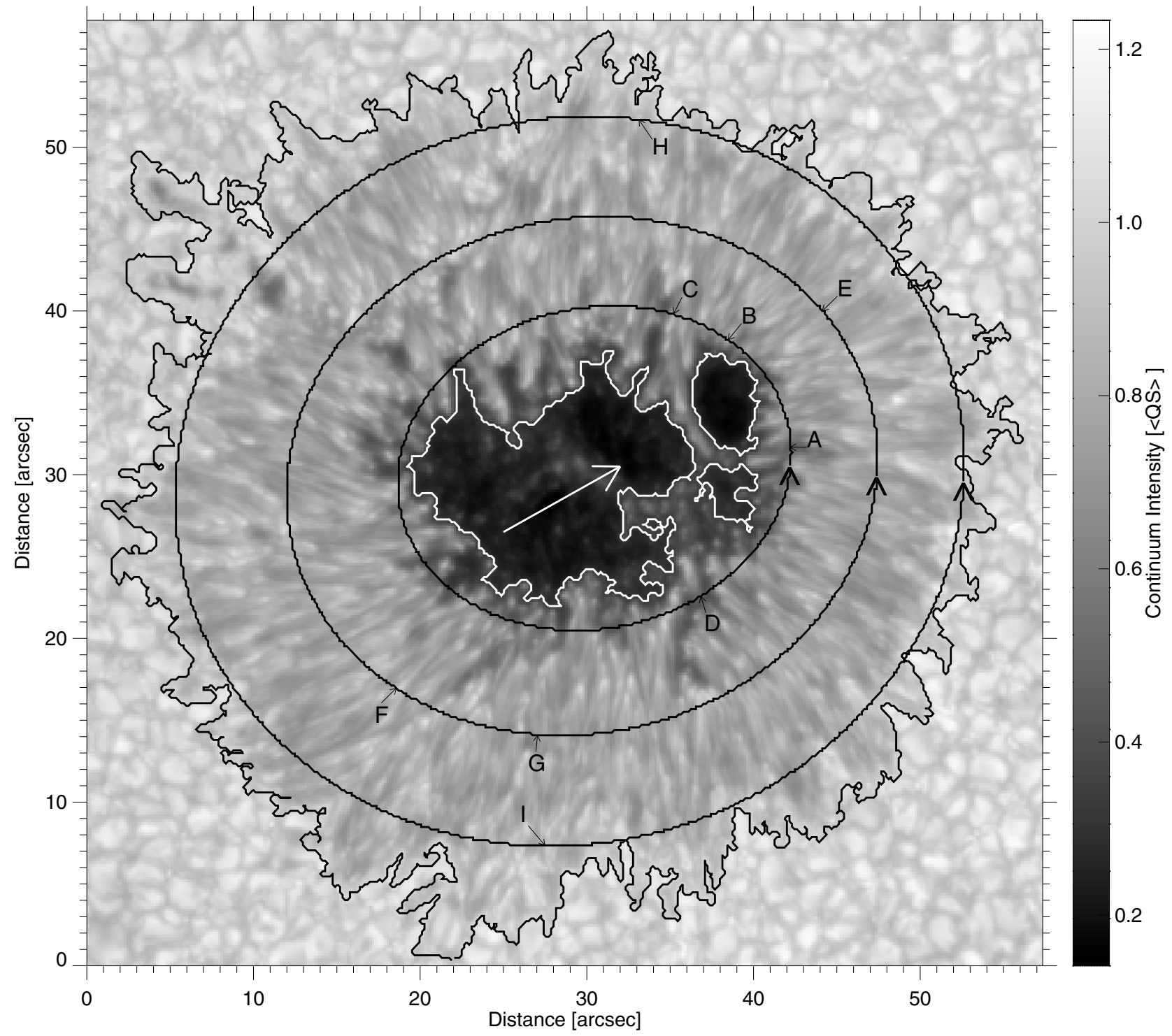

Fig. 4. Map of continuum intensity $\left(I_{c}\right)$ of Spot $\mathrm{D}$ at $\Theta \approx 3^{\circ}$. White/black contours outline the inner/outer penumbral boundary and the arrow points towards disk center. The three ellipses mark tracks in the penumbra at different radii for which $I_{\mathrm{c}}$ and $v_{\mathrm{dop}}$ are investigated in detail in Sect. 4.3. The path is drawn counterclockwise and starts at the tip of the arrow.

is significantly lower in the QS, while the maximum upflow velocity $\left(-3 \mathrm{~km} \mathrm{~s}^{-1}\right)$ is about $50 \%$ higher as in the penumbra.

These results are consistent with a fact mentioned above: Models and other observations imply an overall blueshift of the QS, while our investigation yields a significant redshift of the spatially averaged penumbra.

\subsection{Morphology of velocity field}

The Doppler map of Spot D shows a concentration of upflows in the inner penumbra. They often appear as radially elongated patches next to each other separated by areas without any LOS motion. A typical upflow area is about 2 '. 4 long and 0.5 wide, resulting in a length/width ratio of about 5. Downflows are mainly visible at the penumbra-QS boundary. They are not elongated, but rather roundish. The length of the downflow areas ranges from $1 ., 3$ to $33^{\prime \prime} 0$, while their width extends from 1. .'3 to $2^{\prime \prime} 0$. Thus, with 1 to 1.5 the downflow length/width ratio is less than for the upflow areas. To investigate this morphology quantitatively and to compare the flow field to the continuum intensity, we plotted $v_{\text {dop }}$ and $I_{\mathrm{c}}$ along azimuthal paths.

Figure 6 shows $v_{\text {dop }}$ and $I_{\mathrm{c}}$ in the inner penumbra. What is clearly visible are strong spikes of upflow with velocities of up to $-1.5 \mathrm{~km} \mathrm{~s}^{-1}$. Sometimes downflow peaks are visible, but they are weaker and broader when compared to the upflows. Areas of upflow coincide well with areas of increased $I_{\mathrm{c}}$, and areas of downflow show a reduced $I_{\mathrm{c}}$ (cf. position B \& A in the upper plot of Fig. 6). This agrees with the idea that upflows transport hot plasma from lower layers to the surface, where it cools radiatively and appears bright. The energy release makes it become denser than the surrounding plasma and ultimately sinks back below the surface. On average, the plasma shows an upflow of approx. $-0.2 \mathrm{~km} \mathrm{~s}^{-1}$ in the inner penumbra.

In the mid penumbra (cf. Fig. 7), strong upflow sites are seen less often and downflows become more prominent. Here the penumbra still shows a net upflow of about $-80 \mathrm{~m} \mathrm{~s}^{-1}$, and the correlation between upflow and enhanced $I_{\mathrm{c}}$ is still valid (cf. F \& G in Fig. 7). Surprisingly, the strongest upflow area of Spot $\mathrm{D}$, which is $-2.2 \mathrm{~km} \mathrm{~s}^{-1}$, is located in the middle penumbra. 


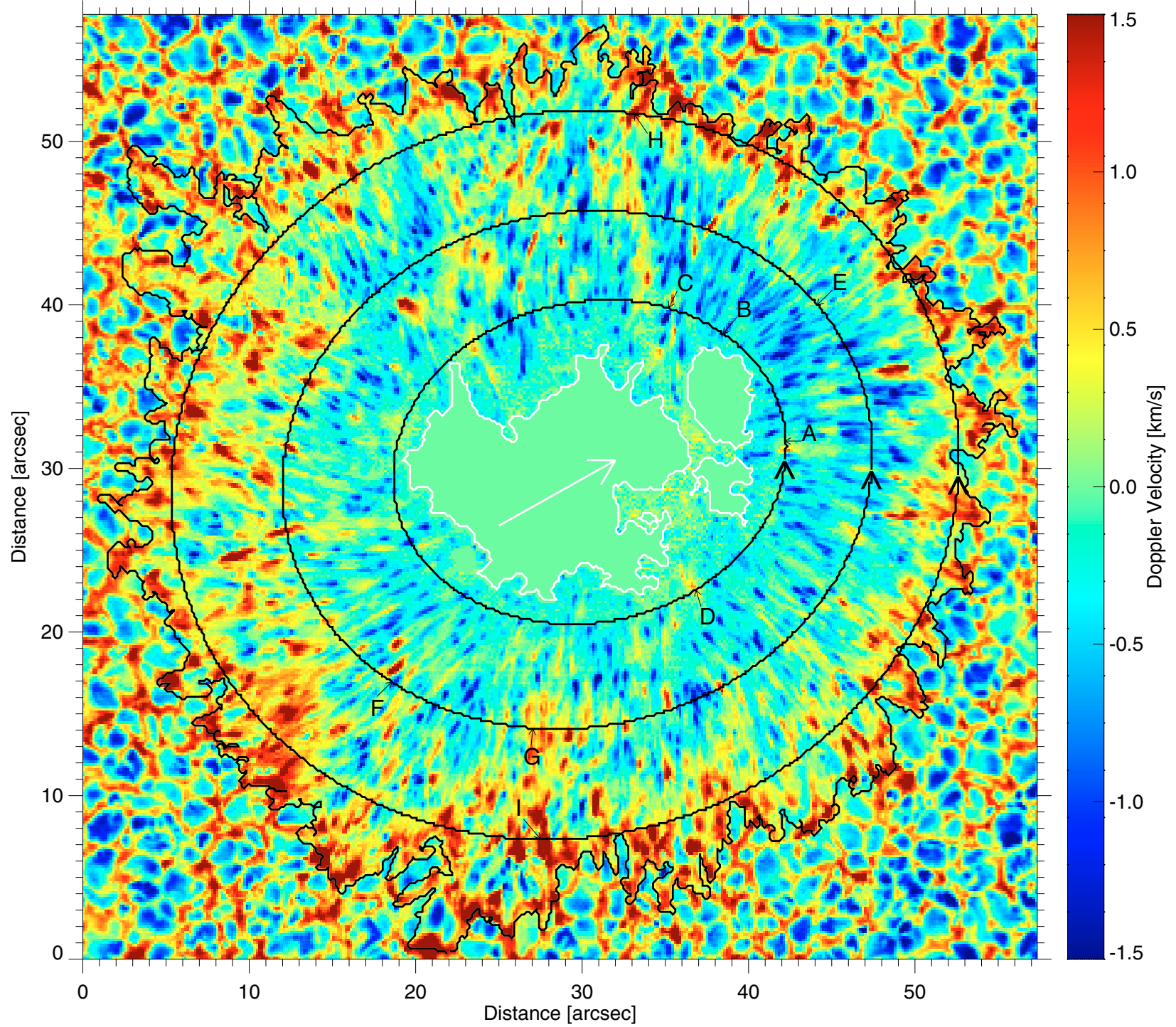

Fig. 5. Same as Fig. 4, but map of LOS velocities in Spot D as derived from the Doppler-shifts in the wing of Fe I $630.15 \mathrm{~nm}$. Upflows are shown from blue to green, downflows are drawn from green to red. The map is saturated at $\pm 1.5 \mathrm{~km} \mathrm{~s}^{-1}$, and the velocities in the umbra are manually set to zero.

With $2.3 \mathrm{~km} \mathrm{~s}^{-1}$, the downflows show velocities of comparable strengths.

In Fig. $8 v_{\text {dop }}$ and $I_{\mathrm{c}}$ of the outer penumbra are plotted. Here strong upflow patches have almost disappeared and the dominant features are downflows, with high velocity amplitudes of up to $3.2 \mathrm{~km} \mathrm{~s}^{-1}$. Not all upflows are within the penumbral boundary, but sometimes belong to granulation. Only one upflow within the penumbra shows velocities of $-1.6 \mathrm{~km} \mathrm{~s}^{-1}$, while the rest is not as strong. On average, the outer penumbra exhibits a downflow of almost $0.45 \mathrm{~km} \mathrm{~s}^{-1}$. Interestingly, there is no distinct correlation between downflows and a decrease in $I_{\mathrm{c}}$ in this part of the penumbra anymore. Position $\mathrm{H} \& \mathrm{I}$ in Fig. 8 indicate two strong downflow places that show an $I_{\mathrm{c}}$ that is larger than $I_{\mathrm{c}}$ of adjacent pixels that exhibit a weaker downflow or even an upflow.

In the inner and mid penumbra, the flow pattern seems to follow the filamentary structure. This is no longer the case in the outer penumbra, where the downflow patches appear roundish and show no filamentary structure. In all azimuthal cuts, no distinct difference between the center and the limb side penumbra can be detected in the flow pattern, as it is the case in penumbrae away from disk center (Tritschler et al. 2004).

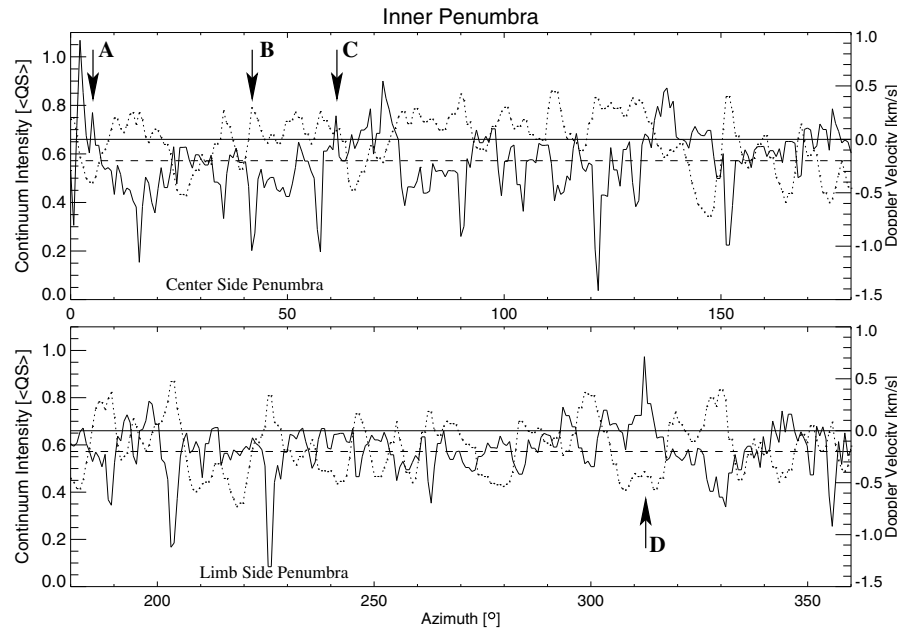

Fig. 6. Continuum intensity (dotted) and Doppler velocity (solid) in the penumbra along the inner path shown in Figs. 4 and 5. Zero velocity is indicated by the solid horizontal line, while the dashed line marks the average velocity along that path. 


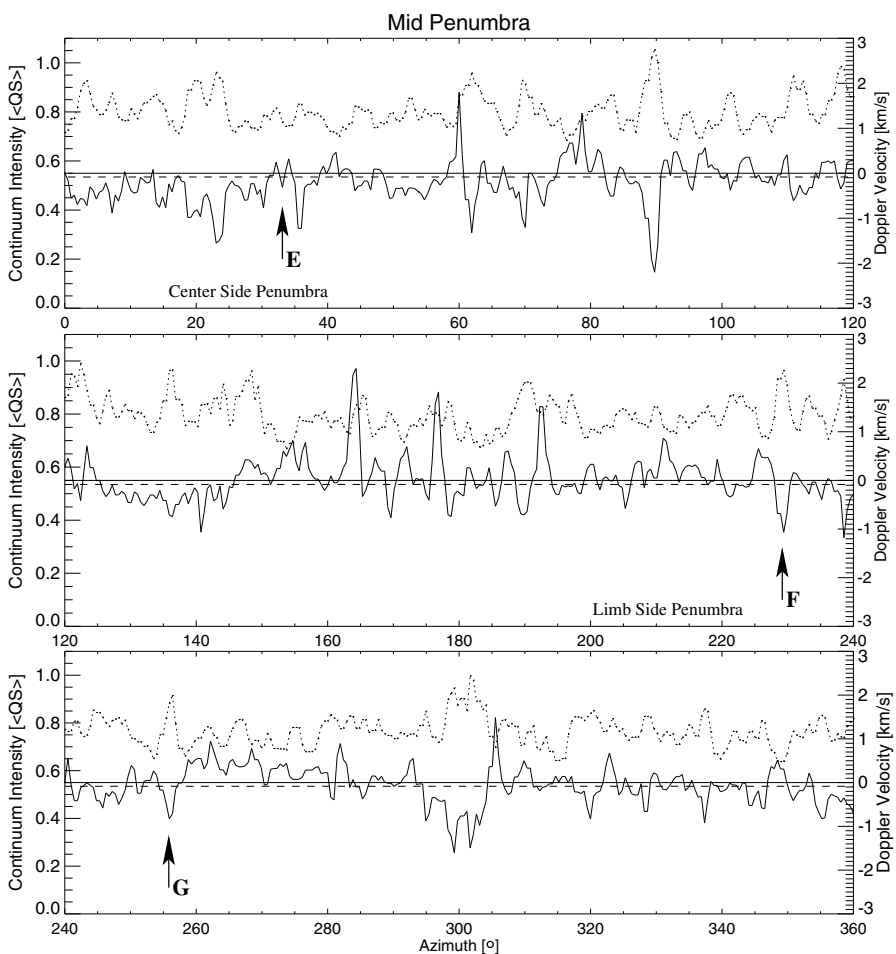

Fig. 7. Same as Fig. 6 but along the central path in Fig. 4.

\subsection{Stokes $V$ signal and magnetized flows}

The Stokes $V$ profile from the downflow region in, e.g. Fig. 2, shows strong asymmetries and more than two lobes. To explain the additional lobes with a simple scenario, we have to assume two magnetic components placed either above or next to each other in the resolution element. In both settings, one of these magnetic components has to be Doppler-shifted (in this example to the red) with respect to the other. Thus, one component would represent a more or less steady magnetized background plasma, while the other component would incorporate strong and magnetized downflows.

The first scenario is more likely as it readily explains the shape of the Stokes I profile (cf. Fig. 2). The line wing is shifted towards the red, while the core is broadened but almost unshifted. Therefore, it is probable that strong velocity gradients along the LOS are present.

Keep in mind that in our case the velocities derived from the line wing of the two iron lines underestimate the flow velocity. Using the bisector to derive Doppler velocities will lead to average flow velocity in the resolution element. Assuming two components - one at rest, the other in motion - would automatically increase the velocities of the latter component.

\subsection{Signatures of roll-type convection?}

In the framework of the gappy penumbral model, energy is transported by roll-type convection in the penumbral gaps, which are areas without magnetic field below $\tau=1$. Thus, upflows would be present in the center or the dark core of the filament, while a downflow should be observable adjacent to it at the position of the lateral brightening (Scharmer \& Spruit 2006). Consequently, a down-up-down flow pattern ought to be visible on a scale of $0.5^{\prime \prime}$ in cuts perpendicular to the filamentary structure of penumbrae.

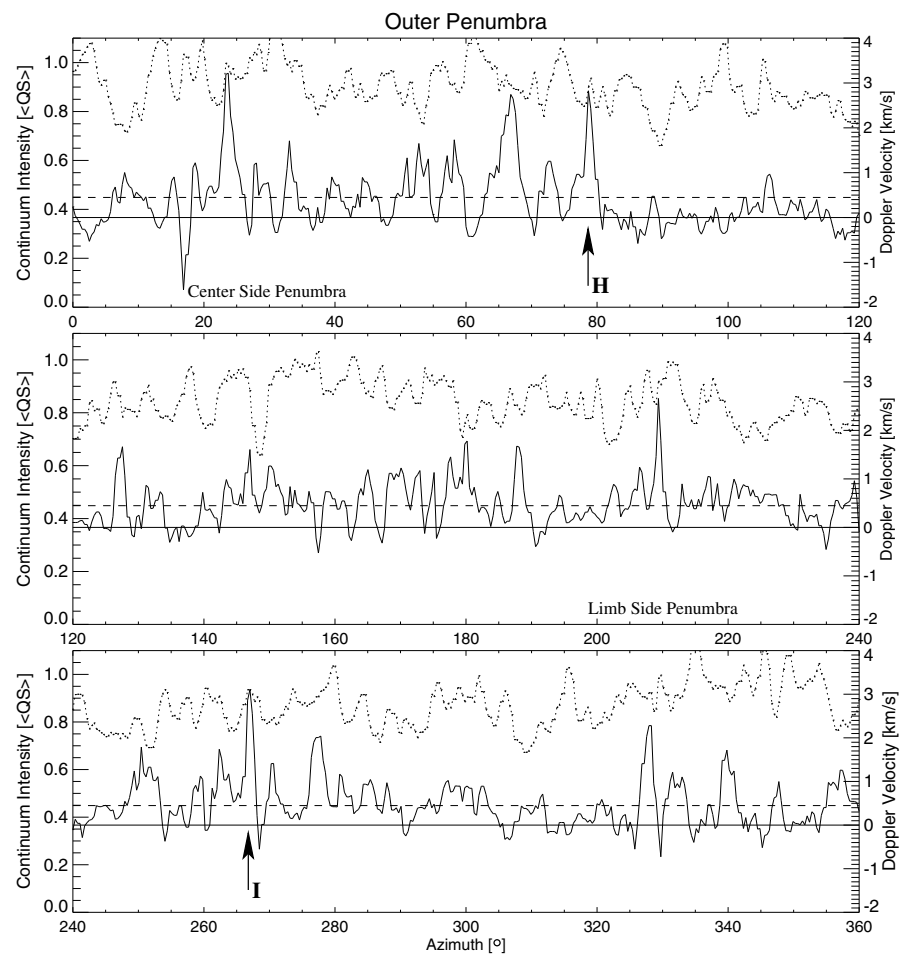

Fig. 8. Same as Fig. 6 but for the outer path in Fig. 4.

The plots of Figs. 6 to 8, however, show that strong up- and down-flow patches have a width of more than $0.5^{\prime \prime}$ and adjacent to them, the flow does not change its direction, but only eases in strength (cf. positions B \& D in Fig. 6). In rare cases, a reversal of the flow direction is observable on a scale of $0.5^{\prime \prime}$ (e.g. positions A \& C in Fig. 6). The depicted flow pattern, however, is just opposite to the signature expected from roll-type convection. One of the very few examples that show a down-up-down flow pattern on a scale of less than $1^{\prime \prime}$ is indicated as E in Fig. 7. The peak-to-peak distance of the downflow patches is $0.6^{\prime \prime}$. A comparison of the maps of $I_{\mathrm{c}}$ and $v_{\mathrm{dop}}$, however, shows that this flow pattern does not coincide with a (dark cored) filament. Other down-up-down flow signatures are observable, but have a width greater than $1^{\prime \prime}$ (e.g. the downflow patches at position F show a peak-to-peak distance of $\left.1.4^{\prime \prime}\right)$. A careful analysis of the other data samples does not yield a down-up-down flow pattern on scales of less than $1^{\prime \prime}$ that could be interpreted as a signature of roll-type convection in penumbral filaments.

\section{Summary and conclusions}

We have presented two independent procedures for calibrating the wavelength scale of Hinode SP data. The first method uses the line core of an average Stokes I profile of the QS. This position was corrected for the CBS of the QS using the results (Beck 2006) of model calculations (Borrero \& Bellot Rubio 2002). In a second approach, we assumed the umbra at rest and determined the center position, which is midways between the two lobes, of umbral Stokes $V$ profiles with an amplitude asymmetry of less than $1 \%$. This center position defines a frame of rest on the solar surface (Rezaei et al. 2006). Within the uncertainties, both methods yield the same results.

To investigate the flow field in several sunspots close to disk center $\left(\Theta \leq 9^{\circ}\right)$, we determined Doppler velocities in the low photosphere by calculating an average bisector in the wings of both Fe lines. Even though in some cases the amplitudes of the 
flows derived from Fe I 630.15 and Fe I 630.25 differ significantly, the morphology of the flow field is unaffected by these differences. Furthermore, we find several prominent examples of multi-lobed Stokes $V$ profiles which can be explained by a magnetized plasma in combination with strong velocity gradience along the LOS. We therefore conclude that the downward component of the EF in the penumbra is magnetized.

Since all the penumbrae we investigated are located close to disk center, we are able to visualize the LOS motion of the plasma normal to the solar surface, but cannot make a proposition considering the horizontal component of the EF. Other investigations (Rezaei et al. 2006), however, show that at large heliocentric angle, the center position of Stokes $V$ profiles is e.g. blueshifted on the center-side penumbra, which indicates a magnetized horizontal component of the EF.

Likewise, we find that the vertical flow pattern in sunspot penumbrae is structured on a small scale. Upflows appear elongated and predominately in the inner penumbra. Downflows concentrate at the penumbra-QS boundary and are rather roundish in shape. $I_{\mathrm{c}}$ and $v_{\text {dop }}$ along azimuthal cuts in the penumbra show that on average there is an upflow present in the inner and midpenumbra, while in the outer penumbra, a downflow is present on average. The upflow patches in the inner penumbra and the downflow locations in the outer penumbra could be interpreted as the sources and the sinks of the EF (Ichimoto \& SOT/Hinodeteam 2009), even though an attribution to individual flow channels is challenging (Ichimoto et al. 2007).

Another finding of our investigation is that the properties of the penumbral flow field are strikingly different from that of the QS. In both iron lines, the area of the QS showing upflows is always larger than the area showing downflows, regardless of the velocity. This is not surprising as the upflows are located in the granules and the downflows are seen in the narrow lanes that form the intergranulum. Interestingly, in the penumbra upflows dominate only at low velocities $\left|v_{\text {dop }}\right| \leq 0.4 \mathrm{~km} \mathrm{~s}^{-1}$. At high velocities $\left|v_{\text {dop }}\right| \geq 0.6 \mathrm{~km} \mathrm{~s}^{-1}$, the area occupied by downflows outnumbers the one showing upflows. This is not only independent of the sunspot sample, but also does not depend on the Fe line we used to derive the Doppler velocity. Furthermore, in all penumbrae upflows with $v_{\text {dop }} \leq-2 \mathrm{~km} \mathrm{~s}^{-1}$ seem to be suppressed, while the velocity of upflows in the QS can reach $v_{\text {dop }}=-3 \mathrm{~km} \mathrm{~s}^{-1}$. On the contrary, downflows with velocities of $9 \mathrm{~km} \mathrm{~s}^{-1}$ are detected in some penumbrae, three times as high as in the downflow regions of the QS. Additionally, we find that on average the penumbra shows a significant redshift corresponding to a downflow of more than $0.1 \mathrm{~km} \mathrm{~s}^{-1}$.

If the penumbral energy transport stems from convective roll motion, a pronounced down-up-down flow pattern should be observable (Scharmer \& Spruit 2006) in azimuthal cuts that cross (dark cored) filaments perpendicularly. In all our data sample, we do not find any indication of a roll-type motion of the plasma on a scale of the width of a filament.

We can speculate on why we do not see such a signature, and various explanation are possible. The easiest interpretation of this observation is that no roll-type convection exists in sunspot penumbrae. On the contrary, one could argue that we do not see hints of roll-type convection due to the limited spatial resolution of the Hinode SP. Another explanation could be that the Fe lines are not affected by the convective plasma motion because it is located only slightly above or entirely below $\tau=1$.

The argument of limited spatial resolution always applies. Observations of (dark-cored) penumbral filaments show that they have a width of less than $1^{\prime \prime}$ sometimes with a dark core around 0.2" in width (Scharmer et al. 2002; Sütterlin et al. 2004; Bellot Rubio et al. 2005). In their MHD simulation of an entire sunspot, Rempel et al. (2009) find a down-up-down flow signature with velocity variation of $\pm 1 \mathrm{~km} \mathrm{~s}^{-1}$ across a filament. Even though the observation of the flow morphology in such darkcored penumbral filaments is at the limit of the spatial resolution of the Hinode SP, a distinct down-up-down flow pattern on scales of $0.5^{\prime \prime}$ should be observable in cuts perpendicular through the filament. If the plasma motion is entirely below $\tau=1$, we have no chance of ever observing it. Yet, any plasma motion below $\tau=1$ will affect the higher atmospheric layers as well, so even if there is convective motion only below the surface, we should see at least a weak Doppler signal in the far wings of the Fe lines.

A more detailed investigation of the flow field in small-scale features of the penumbra, e.g. bright penumbral grains, filaments, will be addressed in a forthcoming publication using the same data. Furthermore, it will be interesting to compare synthetic Stokes profiles computed from realistic simulations of radiative magneto-convection with our observations.

Acknowledgements. We want to thank O. Steiner and R. Rezaei for fruitful discussions, and W. Schmidt for his valuable comments on the manuscript. Part of this work was supported by the Deutsche Forschungsgemeinschaft, DFG project number Schl. 514/3-1. Hinode is a Japanese mission developed and launched by ISAS/JAXA, with NAOJ as domestic partner and NASA and STFC (UK) as international partners. It is operated by these agencies in cooperation with ESA and NSC (Norway).

\section{References}

Auer, L. H., \& Heasley, J. N. 1978, A\&A, 64, 67

Balthasar, H. 1985, Sol. Phys., 99, 31

Beck, C. 2006, Ph.D. Thesis, Albert-Ludwigs-University, Freiburg

Beckers, J. M. 1977, ApJ, 213, 900

Bellot Rubio, L. R. 2009, [arXiv: 0903 . 3619]

Bellot Rubio, L. R., Langhans, K., \& Schlichenmaier, R. 2005, A\&A, 443, L7

Bellot Rubio, L. R., Tsuneta, S., Ichimoto, K., et al. 2007, ApJ, 668, L91

Borrero, J. M., \& Bellot Rubio, L. R. 2002, A\&A, 385, 1056

Centeno, R., Lites, B. W., de Wijn, A. G., \& Elmore, D. 2009, in Proceedings of the Second Hinode Science Meeting, ASP Conf. Ser., ed. M. Cheung et al., [arXiv: 0902.0027]

Dravins, D. 1982, ARA\&A, 20, 61

Heinemann, T., Nordlund, A., Scharmer, G. B., \& Spruit, H. C. 2007, ApJ, 669, 1390

Ichimoto, K., Shine, R. A., Lites, B., et al. 2007, PASJ, 59, 593

Ichimoto, K., \& SOT/Hinode-team 2009, [arXiv : 0903.2605]

Khomenko, E., \& Collados, M. 2007, ApJ, 659, 1726

Lites, B. W., Elmore, D. F., \& Streander, K. V. 2001, in Advanced Solar Polarimetry, ed. M. Sigwarth (San Francisco: ASP), ASP Conf. Ser., 236, 33

Rempel, M., Schüssler, M., \& Knölker, M. 2009, ApJ, 691, 640

Rezaei, R., Schlichenmaier, R., Beck, C., \& Bellot Rubio, L. R. 2006, A\&A, 454, 975

Ruiz Cobo, B., \& Bellot Rubio, L. 2008, A\&A, 488, 749

Scharmer, G. B., \& Spruit, H. C. 2006, A\&A, 460, 605

Scharmer, G. B., Gudiksen, B. V., Kiselman, D., Löfdahl, M. G., \& Rouppe van der Voort, L. H. M. 2002, Nature, 420, 151

Scharmer, G. B., Narayan, G., Hillberg, T., et al. 2008, ApJ, 689, L69

Schlichenmaier, R., Jahn, K., \& Schmidt, H. U. 1998a, ApJ, 493, L121

Schlichenmaier, R., Jahn, K., \& Schmidt, H. U. 1998b, A\&A, 337, 897

Schüssler, M., \& Vögler, A. 2006, ApJ, 641, L73

Solanki, S. K. 1993, Space Sci. Rev., 63, 1

Solanki, S. K., \& Montavon, C. A. P. 1993, A\&A, 275, 283

Spruit, H. C., \& Scharmer, G. B. 2006, A\&A, 447, 343

Sütterlin, P., Bellot Rubio, L. R., \& Schlichenmaier, R. 2004, A\&A, 424, 1049

Tritschler, A., Schlichenmaier, R., Bellot Rubio, L. R., \& the KAOS Team 2004, A\&A, 415, 717

Tsuneta, S., Ichimoto, K., Katsukawa, Y., et al. 2008, Sol. Phys., 249, 167

Vargas Domínguez, S., Rouppe van der Voort, L., Bonet, J. A., et al. 2008, ApJ, 679,900 